\title{
Gibt es ein Leben nach der Arbeit? Zur diskursiven Konstruktion und so- zialen Akzeptanz des „aktiven Alters“
}

Angesichts der prognostizierten Folgen des demografischen Wandels steht die Frage der „Potenziale des Alters“ auf der politischen Agenda weit oben. Im Rückblick auf drei Jahrzehnte öffentlicher Diskurse rund um das Alter lässt sich eine Verschiebung des Fokus auf die aktiven und - zumindest potenziell - produktiven ,jungen Alten“ nachzeichnen. Die Alltagserzählungen älterer Menschen hingegen zeigen eine relative Distanz gegenüber gesellschaftlichen Produktivitätserwartungen. Vorherrschend sind hier, jenseits der empirischen Vielfalt von Sozialfiguren des ,jungen Alters“, Bekundungen einer selbstverständlichen nachberuflichen Aktivität, in der auch ruheständische Momente sowie Motive der Emanzipation von den Anforderungen des Erwerbarbeitslebens ihren legitimen Platz haben.

SILKE VAN DYK, STEPHAN LESSENICH, TINA DENNINGER, ANNA RICHTER

\section{Die gesellschaftliche Neuverhandlung des Alters}

Das Alter - die letzte Phase des um die Erwerbsarbeit herum konstruierten „Normallebenslaufs“ in industriell-kapitalistischen Gesellschaften (vgl. Kohli 1985) - ist in den letzten beiden Jahrzehnten zum Gegenstand eines ungeahnten gesellschaftspolitischen Interesses geworden. Waren die erwerbsarbeitsbefreiten Lebensverhältnisse der Rentnerinnen und Pensionäre mit der Institutionalisierung des materiell gesicherten "Ruhestands“ in der Zeit nach dem Zweiten Weltkrieg zunehmend der gesellschaftlichen Beobachtung entzogen, so rückten sie zuletzt erneut in das Licht der Öffentlichkeit. Hintergrund dieser „Wiederentdeckung" des (höheren) Alters als sozialer Kategorie ist das medial viel zitierte Faktum des demografischen Wandels, das nicht nur in Deutschland Wissenschaft und Politik, Wirtschaft und Gesellschaft zunehmend beschäftigt. ${ }^{1}$

Hinter dem Kürzel „demografischer Wandel“ verbergen sich mindestens zwei analytisch zu unterscheidende, in der öffentlichen Wahrnehmung allerdings eng aufeinander bezogene Wandlungsprozesse. Zum einen ist dies der mittlerweile hinlänglich bekannte Altersstrukturwandel der spätindustriellen Gesellschaften, der durch die Kombination von sinkender bzw. stabil niedriger Fertilität einerseits und über den gesamten Lebenszyklus ebenso niedriger und weiter sinkender Mortalität andererseits längerfristig zu einer
Zunahme des Anteils älterer Menschen an der Gesamtbevölkerung führt (vgl. z. B. Schimany 2003). Zum anderen aber vollzieht sich, parallel dazu, ein in der Sozialgerontologie als Strukturwandel des Alters bezeichneter Prozess der Veränderung der „letzten Lebensphase“ selbst, zu welchem neben dem bereits genannten Aspekt der Hochaltrigkeit (also der steigenden Lebenserwartung nachrückender, aber auch bereits lebender Alterskohorten) die weiteren Dimensionen der Feminisierung und Singularisierung (im Sinne der statistischen Dominanz von Frauen und Einpersonenhaushalten), der Entberuflichung (im Lebenslauf zunehmend früher vollzogene Erwerbsentpflichtung) sowie der Verjüngung des Alters gezählt werden (vgl. Tews 1990).

Dass die heutigen Alten ,jünger" sind als jene früherer Zeiten, also etwa ein heute 60-jähriger Mensch nicht die Alterssymptome einer gleichaltrigen Person seiner bzw. ihrer Elterngeneration aufweist, dürfte zum allgemein geteilten gesellschaftlichen Wissensbestand in Bezug auf das Alter der Gegenwart gehören. Und ,jünger“ bezieht sich

\footnotetext{
1 Ausführlich beschäftigt sich mit dieser gesellschaftlichen Neubewertung des Alters die im Herbst 2013 erscheinende Monografie "Leben im Ruhestand“ (Denninger et al. 2013), die neben einer umfassenden theoretisch-methodologischen Fundierung unseres alterssoziologischen Forschungsprogramms eine unkonventionelle, unterschiedliche Textgattungen einbeziehende Darstellung der hier nur skizzierten empirischen Forschungsergebnisse enthält.
} 
hier nicht nur auf die Alterskategorie im engeren Sinne, sondern umschreibt ein ganzes Set an Eigenschaften, das heutige „Alte“ zu Noch-Nicht-Alten (im Lebenszyklus wie auch in der Selbstwahrnehmung) bzw. Nicht-Mehr-Alten (nämlich im intergenerationalen Vergleich) werden lässt: „Die heutigen Seniorinnen und Senioren sind im Durchschnitt gesünder, besser ausgebildet und vitaler als frühere Generationen.“ (Deutscher Bundestag 2010, S. 5) Was läge daher in Zeiten, da soziale (Dienst-)Leistungen reduziert und Risiken sozialpolitisch individualisiert werden, näher, als dieses „Nicht-Alter“ der "Alten“ als ausbeutbare Ressource zu entdecken? Was läge näher als auf den Gedanken zu kommen, dass diese „Alten“ zur Bearbeitung der Folgen jenes demografischen Wandels herangezogen werden könnten, dessen Ausdruck ihre zunehmende Zahl ist? Oder anders gesagt: dass der Strukturwandel des Alters zur Bewältigung des Altersstrukturwandels beitragen könnte?

Genau dieser Gedanke ist im Laufe der beiden letzten Jahrzehnte zur Leitidee des politisch-sozialen Umgangs mit dem Alter und der "gesellschaftlichen Alterung " geworden. "Averting the old age crisis“ (World Bank 1994) - womit insbesondere die Abwendung einer Krise der bei steigender Lebenserwartung und sinkender Erwerbstätigenzahl tendenziell stärker in Anspruch genommenen Alterssicherungssysteme gemeint ist - mithilfe der „jungen Alten“ (vgl. van Dyk/Lessenich 2009) selbst: So lautet die gängige alterspolitische Zukunftsformel der Gegenwart. Auf europäischer Ebene seit Ende der 1990er Jahre in Gestalt der EU-offiziellen Politik des „Active Ageing“ vorangetrieben (vgl. Boudiny 2012) und auf nationaler Ebene in eine umfassendere programmatische Bewegung zum ,aktivierenden“ Umbau des Sozialstaats eingebettet (vgl. Lessenich 2008), steht die Mobilisierung der "Potenziale des Alters“ (vgl. Deutscher Bundestag 2006; so auch Grabka in diesem Heft) auf der Agenda der ,alternden Gesellschaft" mittlerweile ganz oben.

Was diese Mobilisierungsstrategie nach dem Willen der Akteure im alterspolitischen Feld besonders attraktiv machen soll, ist ihr öffentlich reklamierter Charakter als „win-win“Strategie: „The beauty of this strategy is that it is good for everyone." (Walker 2002, S. 137) Nicht nur die Alten - als Nicht-Alte -, so heißt es, würden davon profitieren, indem ihnen Möglichkeiten zum Einsatz ihrer Fähigkeiten etwa im Rahmen bürgerschaftlichen Engagements eröffnet werden und sie in Folge der Demonstration ihrer Leistungsfähigkeit in den Genuss der gesellschaftlichen Aufwertung des höheren Lebensalters kommen. Auch „der Gesellschaft“ als Ganzes komme der so geartete Einsatz des Humanvermögens ,jungalter" Menschen unmittelbar zugute. Tatsächlich aber sind win-win-Konstellationen in einer von Machtverhältnissen und Interessenkonflikten durchzogenen Gesellschaft äußerst selten. Und deshalb gilt es zu fragen: Welche Akteure propagieren Altersaktivität als gesellschaftspolitische Zauberformel? Was meint „aktives Alter(n)“ in diesem Zusammenhang? Und vor allem: Was wissen wir eigentlich über die Haltungen und Wünsche der zur Aktivität aufgerufenen Älteren selbst?
Im Rahmen des Forschungsprojektes „Vom, verdienten Ruhestand' zum ,Alterskraftunternehmer?" sind wir dem Wandel von Bildern wie auch von Praktiken des Alters in der Bundesrepublik Deutschland empirisch nachgegangen. ${ }^{2}$ Zum einen wurde auf der Basis eines breit angelegten Textkorpus über einen Zeitraum von fast drei Jahrzehnten hinweg die öffentliche Neuverhandlung des Alters rekonstruiert, zum anderen interessierte sich das Projekt dafür, ob sich in der gesellschaftlichen Gegenwart auch schon eine empirisch rekonstruierbare soziale Realität „aktiver Alter“ - also eine lebenspraktische Umsetzung jener Neuverhandlung - finden lässt. Im Folgenden skizzieren wir kurz das soziologische Anliegen des Projekts (Abschnitt 2), um sodann zentrale Befunde seiner beiden Bestandteile - der Analyse des Wandels politisch-medialer Altersdiskurse (3) sowie der qualitativen Befragung ,junger“ Alter selbst (4 und 5) darzulegen. Abschnitt 6 zieht ein knappes Fazit.

\section{Vom verdienten Ruhestand zum Alterskraftunternehmer?}

Theoretisch und methodologisch schließt unser Vorhaben an die seit einem Jahrzehnt auch im deutschen Sprachraum etablierte Gouvernementalitätsforschung an (vgl. Krasmann/Volkmer 2007). An den Programmen und Technologien der Menschenführung und der politischen Konstitution selbstführender Subjekte interessiert, hat dieser am Spätwerk Michel Foucaults orientierte Forschungsstrang einen erhellenden diagnostischen Blick auf die ökonomisierende Regierung des Sozialen im „neoliberalen“ Zeitalter eröffnet (vgl. z. B. Bröckling et al. 2000). Die Analyse der Neuverhandlung von Alter und Ruhestand profitiert von einer gouvernementalitätstheoretischen Fundierung in besonderem Maße, vollziehen sich die aktuellen Versuche der Re-Regulierung des Alters doch nicht in erster Linie als erzwungener Prozess - als formale Verpflichtung -, sondern als moralischer Appell qua Anleitung zur Selbstführung.

Allerdings meiden die diesem Ansatz zuzuordnenden Arbeiten systematisch die empirische Überprüfung der Effekte der von ihnen (in Gestalt von Lehrbüchern, Ratgeberliteratur, Gesetzestexten usw.) untersuchten Regierungsprogramme und -technologien auf die sozialen Lebenswelten und Alltagspraktiken ihrer Adressaten. „Die Vermessung

\footnotetext{
2 Das Projekt wurde im Rahmen des Sonderforschungsbereichs 580 "Gesellschaftlicher Wandel nach dem Systemumbruch" an den Universitäten Jena und Halle-Wittenberg durch die Deutsche Forschungsgemeinschaft gefördert. Entsprechend waren in ihm auch die innerdeutschen Ost-West-Differenzen mit Blick auf das "aktive Alter(n)“ Thema. Sie stehen die allerdings nicht im Zentrum des vorliegenden Beitrags; vgl. zu diesem spezifischen Fokus Denninger et al. (2012)
} 
gouvernementaler Kraftfelder erlaubt keine Aussagen darüber, wie Menschen sich tatsächlich in ihnen bewegen.“ (Bröckling/Krasmann 2010, S. 28) Diese forschungsprogrammatische Beschränkung auf Programmanalysen schien uns soziologisch unbefriedigend zu sein: Denn dass die aus gouvernementalen Texten herausgelesenen Führungsregime wirken, wird hier schlicht theoretisch (voraus)gesetzt - was auch immer die „Subjekte“ tun, ob sie sich nun passiv unterwerfen, kreativ handeln oder aber aktiv widerstehen, stets sind sie ein Produkt des jeweils herrschenden Regierungsregimes. Uns hingegen interessierte gerade die im engeren Sinne gesellschaftliche Seite der Medaille: das je historisch-konkrete Anwendungs-, Aneignungs- und Anverwandlungshandeln der diesen Regimen ,unterworfenen“ Subjekte, also gewissermaßen die soziale Akzeptanz der nur durch ihr Handeln hindurch „herrschenden“ Regierungsprogramme und -technologien (vgl. Denninger et al. 2010).

Unser Interesse an der gesellschaftlichen Vermittlung von politischen Programmen bzw. ihren Ausdeutungen, Aneignungen und „Umschreibungen“ begründet ein zweigliedriges Forschungsdesign: Die Analyse der programmatischen, politisch-medialen, aber auch wissenschaftlichen Neuverhandlung des Alters seit Anfang der 1980er Jahre bis in die Gegenwart $^{3}$ wird ergänzt durch die Durchführung qualitativer, problemzentrierter Interviews mit der Zielgruppe dieser Programmatiken, den sogenannten ,jungen Alten“.

Mit der Analyse der Wissensbestände, wie sie sich im öffentlichen Diskursgeschehen rund um die gesellschaftliche Verhandlung von Altersbildern herauskristallisiert haben, ist jedoch nur ein Element des uns interessierenden "Altersdispositivs" in den Blick genommen, nämlich das epistemische: das öffentlich konstruierte und legitimierte Bild davon, was „das Alter“ ist (und was nicht) bzw. wie „alte Menschen“ sind (und wie nicht). Wir begreifen das Dispositiv darüber hinausgehend mit Foucault als eine Verknüpfungsordnung heterogener Elemente, zu denen auch Institutionen bzw. institutionelle Programme, Objekte und Artefakte, Alltags- und Körperpraktiken gehören (vgl. Denninger et al. 2010, S. 217ff.). Ziel unserer Untersuchung war es, gerade die spezifischen - und wechselnden - Verschränkungen von öffentlichen Wissensbeständen mit diesen weiteren Elementen aufzuzeigen.

\section{Die Neuverhandlung des Alter(n)s: vom Ruhestands- zum Produkti- vitätsdispositiv}

Die die Neuverhandlung von Alter und Ruhestand strukturierenden Dispositive können verstanden werden als „Erzählungen der sozialen Wirklichkeit [...], durch die Elemente aus vielen unterschiedlichen Gebieten miteinander verknüpft werden und die dabei den Akteuren ein Set symbolischer Assoziationen an die Hand geben, die so ein geteiltes Ver- ständnis suggerieren“ (Hajer 1997, S. 113) - ein geteiltes Verständnis davon zum Beispiel, wie das Alter ist und was es ausmacht, was alte Menschen tun und lassen, wie sie leben und sich geben. Diese Verknüpfungen aktualisieren und konstituieren, rationalisieren und materialisieren ein gesellschaftliches Wissen von dem und über das Alter, das aus unterschiedlichsten Quellen der sozialen Welt und des gesellschaftlichen Lebens zusammenfließt und das doch immer anfällig für „Störungen“ und „Fehlaneignungen“ bleibt. Auf der Grundlage unseres empirischen Materials haben wir drei verschiedene gesellschaftliche Altersdispositive identifizieren können, die einer gewissen zeitlichen Chronologie folgen, im historischen Verlauf aber weniger einander ablösen als sich vielmehr überlagern und miteinander interagieren.

Bis in die 1980er Jahre hinein findet sich in der deutschen Öffentlichkeit das verankert, was seither zunehmend als negative Hintergrundfolie für die Etablierung neuartiger Dispositivkonstellationen fungiert: das Dispositiv des „ruheständischen Alters“. Ist vom Alter die Rede, dann verschränken sich im Ruhestandsdispositiv das Wissen um dessen Erwerbsentpflichtung und materielle Versorgung (über die Institution der gesetzlichen Rentenversicherung) mit Assoziationen des Rückzugs aus dem öffentlichen Leben und der sozialen Vereinzelung, des körperlichen Abbaus und des Nachlassens von außeralltäglichen Aktivitäten. Die Kurzerzählung eines gesicherten und weitgehend sorglosen, aber tendenziell auch sinnentleerten und wenig freudvollen Alters verknüpft - in wechselnden Kombinationen und mal kürzeren, mal längeren Verkettungen - narrative Elemente wie die Rente und den Kuraufenthalt, Haus und Garten, Sofa und Fernseher, Corega Tabs und Altenheim zu einem Bild des „Ruhestands“, dem irgendwie etwas Gestriges anhaftet, und dies zumal im Lichte neuerer Wissensbestände, die dieses Bild historisch zunehmend überformen und damit nicht nur empirisch, sondern auch normativ infrage stellen.

So lässt sich seit Mitte der 1980er Jahre eine neue dispositive Ordnung ausmachen, die in dem Kunstwort des „Unruhestands“ ebenso beredten wie sinnhaften Ausdruck findet. Es sind einerseits wissenschaftliche Wissensbestände, die in das neu entstehende Bild einer ,unruheständigen“ Lebensführung im Alter eingehen, nämlich das Wissen

3 Diese Analyse beruht auf ca. 2.500 Textdokumenten aus den Jahren 1983 bis 2011, die ein breites Spektrum an Publikationen aus dem politisch-medialen Raum umfassen von Tageszeitungen (FAZ, taz, BILD, Neues Deutschland) über Zeitschriften (SPIEGEL, Brigitte und Brigitte Woman, Superillu, Apotheken Umschau) bis hin zu politischen bzw. wissenschaftlich-politischen Medienerzeugnissen (Altenberichte der Bundesregierung, Selbstbeschreibungen altenpolitischer Modellprogramme, Parteiprogramme usw.) und Gesetzestexten.

4 Die Interviews wurden 2009 und 2010 mit insgesamt 55 älteren (60- bis 72-Jährigen), verrenteten Männern und Frauen unterschiedlicher Bildungs- und Einkommenskonstellationen in Jena und Erlangen geführt. 
um die Plastizität des alten Körpers - einschließlich des alternden Hirns - und um die zahlreichen Kompetenzen des Alters, um die sich ein Normativ des eigentätigen Funktionserhalts rankt: Die Alten werden dazu ermuntert, sich um eine gesunde Lebensführung, um Fitnesstraining und Gehirngymnastik, sprich, um sich selbst zu kümmern - im Interesse einer subjektiv erfüllten und individuell „erfolgreichen" Langlebigkeit, aber auch um die Gesellschaft von den Kosten eines „siechen“ Alters zu entlasten. Hier findet sich bereits in der Figur des Unruhestandes ein erstes produktivistisches Moment. Wer rastet, der rostet - und wer sich bewegt, altert langsamer bzw. bleibt im Alter länger jung. In diese Erzählung von den neuen Möglichkeiten, sich selbst als ,jung-alter“ Mensch zu konstituieren und durch Aktivität zu immer neuer bzw. erneuerter Lebenskraft zu finden, gehen neuartige Elemente ein, die sich von denen der überkommenen und zunehmend der distanzierenden Abgrenzung dienenden Ruhestandserzählung signifikant unterscheiden: etwa das Radfahren und der Hometrainer, die Walking-Stöcke und der Computer, die Fernreise und der Jakobsweg, die Alten-WG und das Seniorenstudium. Jeder dieser Begriffe ruft in der öffentlichen Kommunikation Bilder des Alters auf - oder bringt sie erst hervor -, die im Wortsinne „un-ruheständischer" kaum sein könnten.

Doch auch dieses Dispositiv wird seit Ende der 1990er Jahre und verstärkt seit Mitte des vergangenen Jahrzehnts durch neue dispositive Verknüpfungen überlagert, die unruheständlerische Momente zugleich fortführen und verschieben: das Dispositiv des „Produktiven Alters“ gewinnt seither an Bedeutung. Sprechend für diese Ordnung - und kennzeichnend für ihre Genese im politischen Feld - ist eine institutionelle Kampagne des Bundesseniorenministeriums aus dem Jahr 2009, die unter dem Slogan „Zähl Taten, nicht Falten“ alte Menschen dazu aufforderte, sich bürgerschaftlich zu engagieren- und damit nicht nur für sich selbst, sondern auch für andere aktiv zu werden. Darin liegt denn auch der entscheidende Unterschied des „Produktivitäts“zum früheren „Unruhestands“-Dispositiv des Alters: Die Kompetenzen, die den unruheständisch jung gebliebenen Alten hier zugeschrieben werden, gelten als Potenziale eines produktiven Tätigwerdens zum Nutzen der Allgemeinheit und begründen damit den zumindest impliziten Aufruf, durch Realisierung dieser Potenziale sozial verantwortlich zu handeln. ${ }^{5}$ In diesem Sinne werden hier andere Elemente erzählerischer Konstruktion prominent - zu nah sind selbst Hometrainer, Walking-Stöcke oder Seniorenstudium, als Objekte und Institutionen des Unruhestands, an dem Bild einer zwar eigenverantwortlichen, aber eben auch eigennützigen, selbstbezüglichen Lebensführung, zu weit entfernt hingegen von der öffentlichen Zurschaustellung gesellschaftlicher Verantwortung im Alter. Im Sinne des „Produktiven Alters“ muss es nun schon die „Rente mit 67“ (als Verlängerung des Erwerbslebens zwecks Entlastung der Alterssicherungssysteme), das Bürgerschaftliche Engagement (als freiwilliger Dienst an der Allgemeinheit) oder die Tätigkeit als „Senioren-Trainer bzw. Trainerin“ (sprich als
Ausbilder anderer Älterer zu bürgerschaftlich Engagierten) sein, um aus den aktiven Alten auch im produktiven Sinne "gute“ Alte zu machen: nützliche, wertschaffende und ertragreiche, damit aber auch erträgliche und akzeptierte Mitglieder der Gesellschaft - was sich heute, so lernen wir auf diese Weise, durchaus nicht mehr von selbst versteht.

\section{Alter und Ruhestand aus Sicht der Interviewten}

Vieles ließe sich über die skizzierte historische Dynamik gesellschaftlicher Altersdispositive an dieser Stelle sagen: über die bemerkenswerte Delegitimierung des „Ruhestands“ als Lebensform in der öffentlichen Kommunikation; über die hochgradig selektive Zuweisung von Kompetenzen an die „jungen“ Alten (denen immer wieder Ruhe, Gründlichkeit, Erfahrung zugeschrieben wird, nie aber Initiative, Innovativität, Flexibilität - die durchgängig Attribute der „wirklich“ Jungen bleiben); oder über die sozialstrukturelle Schlagseite der Erzählung vom ,jungen“, aktiven und produktiven - oder genauer: potenziell produktiven - Alter, die tendenziell den sozialen Gepflogenheiten und normativen Standards der gehobenen bürgerlichen Sozialmilieus entspricht. ${ }^{6}$

Im Kern interessierte uns jedoch die Frage nach der Reproduktion bzw. Transformation der neuerdings aktivisch-produktivistisch geprägten Ordnung des Alter(n)s durch die und in den alltäglichen Deutungen und Praktiken älterer Menschen selbst. Wie beschreiben also die von uns befragten Älteren ihr Leben nach dem Ende des Erwerbslebens? Welche Orientierungen rahmen ihre Alltagserzählungen? In welcher Weise werden die identifizierten Dispositive verarbeitet - oder aber gerade nicht?

Zunächst ist auffällig, dass das Ruhestandsdispositiv weiterhin den zentralen Orientierungsrahmen fast aller Interviewten darstellt - sei es als negative Abgrenzungsfolie oder als positive Bezugsgröße, nur in Ausnahmefällen spielt es gar keine Rolle. In den allermeisten Fällen wird der Über-

5 Vgl. dazu auch Lessenich 2013. Bemerkenswert in diesem Zusammenhang sind sowohl die Bebilderung der entsprechenden Plakatserie - mit gut aussehenden, die Kraft von mindestens zwei Herzen ausstrahlenden Alten - wie auch die erwerbsgesellschaftlichen Konnotationen und geschlechterspezifischen Codierungen der amtlich beworbenen Tätigkeiten: Während ein 82-jähriger (!) ehrenamtlicher Unternehmensberater mit der Aktivitätsbeschreibung „Er hat 12 Manager beraten, 5 Existenzen aufgebaut und 3 Pleiten verhindert. In der letzten Woche." vorgestellt wird, heißt es bei einer 83-jährigen (!) ehrenamtlichen außerschulischen Pädagogin: „Sie hat 8 Männer geliebt, getröstet, gescholten und ihnen vergeben. Alles diese Woche."

6 Womit sich "active ageing" bei genauerem Hinsehen als eine klassenpolitische Distinktions-, Behauptungs- und Herrschaftsstrategie erweist (vgl. Graefe/Lessenich 2012). 
gang in die Rentenphase als ein befreiendes Ereignis wahrgenommen, die Nacherwerbsphase als eine Zeit der persönlich-souveränen Ausgestaltung des Lebensalltags dargestellt. Länger oder gar „ausschlafen“, ausgiebig (und gemeinsam) frühstücken, Zeitung lesen: das sind für die Älteren durchweg die alltäglichen Insignien ihrer „späten Freiheit“" (Rosenmayr 1983). Die wiederkehrende Betonung des Ruhestands als Zeit der Selbstbestimmung ist augenfällig und kontrastiert mit der im jüngeren Aktivierungsdiskurs dominanten Idee einer Selbststeuerung Älterer im Sinne der Zielperspektive produktiven Engagements. Im Vordergrund steht für viele der Befragten hingegen eine (wie auch immer unbestimmte) Vorstellung von Autonomie im Sinne selbsttätiger Zielsetzung - eine Form der alltäglichen Lebensgestaltung, die für die Befragten zu Zeiten der Erwerbstätigkeit in aller Regel nicht praktizierbar war.

So ungebrochen die Verankerung des Ruhestands als legitime und finanziell abgesicherte Erwerbsentpflichtung ist, so sehr dient fast allen Interviewten ein negatives Bild des Ruhestandsalltags - subjektiv mit Passivität und Langeweile verbunden - als Abgrenzungsfolie. Hinter der gewonnenen Freiheit lauert die von den Interviewten des Öfteren als solche benannte „Gefahr“, dass man sich im Alter „gehen lässt“ und „abgleitet“. Interessanterweise spielt deshalb neben dem Topos der Selbstbestimmung jener der Selbstverpflichtung eine zentrale Rolle in den (Selbst-)Darstellungen der Befragten. Doch auch hier geht es mehrheitlich nicht um die Verpflichtung auf Vorstellungen eines Produktiven Alters, sondern vielmehr um die persönliche Bewältigung eines nach der Erwerbsarbeit ungewohnt unstrukturierten Alltags - ein lebenspraktischer Aspekt des „aktiven Alters", der in der öffentlichen Diskussion weitgehend ausgeblendet bleibt.

Unsere Interviews offenbaren zudem komplexe Verflechtungen der die Nacherwerbsphase gleichermaßen prägenden Normen der Aktivität einerseits, der Ruhe andererseits. Als „aktiv" schildern sich fast alle Befragten, Aktiv-Sein gilt den Älteren durchgängig - und unabhängig von ihrem Aktivitätsgrad - als etwas Gutes und Wünschenswertes. Das SelbstBewusstsein einer vom medizinisch-gesundheitlichen Fortschritt profitierenden Kohorte („wir sind ja viel länger fitter“) zieht sich praktisch durch alle Interviews, eine vita activa gilt als Teil der conditio humana, von der die jungen Alten nicht (warum auch?) ausgenommen sind. Mit einem produktivistischen Verständnis von Aktivität hat dies zunächst wenig zu tun, von einer bedeutsamen Ausnahme abgesehen: Die von vielen Befragten für wichtig erklärte Prävention vorzeitigen Alter(n)s durch Aktivität und Bewegung wird nicht nur mit dem individuellen Gewinn längerer beschwerdefreier Lebenszeit verbunden, sondern begegnet uns zudem als moralisch grundierte Verpflichtung, sich „gesund zu halten“, um die Krankenversicherung finanziell nicht zu belasten. Das weite Aktivitätsverständnis der vita activa verschränkt sich hier mit Elementen aus dem Unruhestandsdispositiv, die eine eigenverantwortliche „Schadensminderungspflicht“ gegenüber der Gesellschaft postulieren.
Wie stark die Norm der (fortgesetzten) Aktivität wirkt, sehen wir nicht zuletzt daran, dass das kommunizierte Aktivitätsniveau nicht selten in auffälliger Weise mit der tatsächlichen Lebensführung kontrastiert, ein in der Gerontologie seit Langem als „busy ethic“ (Ekerdt 1986) bekanntes Phänomen: Diese ermöglicht es Menschen in der Nacherwerbsphase, das eigene Leben in der Selbstdeutung und -darstellung an die Alltagserfahrungen, Sozialnormen und Rechtfertigungsmuster des erwerbstätigen Erwachsenenalters anzuschließen und damit den biografischen Bruch zu kitten, den der Erwerbsausstieg zwangsläufig bedeutet. Gleichwohl gilt: Die meisten Befragten, selbst diejenigen, die ein hohes Maß an Geschäftigkeit bekunden, betonen durchaus die Bedeutsamkeit einer altersspezifischen Ruhe. Neben der üblichen Abwehr eines passiven Ruhestandsalltags findet sich daher oftmals auch die spiegelbildliche Ablehnung einer als geradezu manisch empfundenen „Hyperaktivität" anderer Älterer.

\section{Die vielen Welten des Nach- erwerbslebens}

Der von unseren Befragten durchweg geäußerte positive Bezug auf „Aktivität“ im Alter ist zugleich so deutungsoffen, dass sich mit ihm ein klassischer „Lebensabend“ mit Hausund Gartenarbeit, Enkelkinderbetreuung und Kegelgruppe subjektiv ebenso gut verbinden lässt wie ein mit politischem und ehrenamtlichem Engagement oder unterschiedlichsten Bildungsaktivitäten angefüllter „Zweiter Frühling“. Neben der Rekonstruktion der Gemeinsamkeiten der Interviews mit Blick auf die Dispositive des Ruhestands, Unruhestands und Produktiven Alters haben wir deshalb eine "Sortierung“ entlang dreier zentraler Analysedimensionen vorgenommen:

- der Nacherwerbsorientierung der Interviewten, die vor dem Hintergrund der drei Dispositive vermessen wird,

- der erzählten Praxis (d. h. der alltagspraktischen Realisierung dieser Orientierung) sowie

- der Positionierung zur produktivistischen Neudeutung des Alters. ${ }^{7}$

\footnotetext{
7 Zum Ende jedes Interviews wurde eine Meinungsäußerung zu folgendem Passus aus einem Flyer der Bundesregierung zum Sechsten Altenbericht erbeten: „Bei aller Verantwortung der Gesellschaft darf aber nicht übersehen werden, dass die Rechte des einzelnen Menschen mit Verpflichtungen gegenüber der Gemeinschaft einhergehen. Insgesamt sind die heute älteren Menschen im Vergleich zu früheren Generationen gesünder, sie verfügen über einen höheren Bildungsstand und über bessere finanzielle Ressourcen. Nach Auffassung der Kommission leitet sich daraus die Verpflichtung ab, vorhandene Ressourcen verantwortungsvoll einzusetzen."
} 
Die aus den je unterschiedlichen Kombinationen dieser drei Merkmale sich ergebenden empirischen Muster in dem von uns untersuchten Sample sind weder im Sinne einer abschließenden Zuordnung von „Fällen“ zu „Typen“ zu verstehen, noch sollen sie einem - qualitativen Untersuchungsdesigns eher fremden - Bedürfnis nach Darstellung von Häufigkeitsverteilungen Ausdruck geben bzw. Vorschub leisten. Vielmehr verweisen sie auf charakteristische Merkmalskonstellationen, die einen strukturierten Einblick in die soziale Vielfalt des Lebens "nach der Arbeit“ erlauben, ohne deshalb Strukturierungen nach anderen Kriterien entgegenzustehen. ${ }^{8}$ Im Folgenden wird ein analytisches Schlaglicht auf sechs - in diesem Sinne „typische“ - Sozialfiguren des Nacherwerbslebens geworfen.

(1) Der zufriedene Ruheständler hat ein durchweg positives Ruhestandsverständnis: Die Idee der Entpflichtung ist für ihn nicht auf die Befreiung von Erwerbsarbeit beschränkt, sondern zielt auf das Nacherwerbsleben selbst, das als verdiente Ruhe begriffen wird. Kennzeichnend ist hier eine starke Orientierung auf die Familie und die häusliche Sphäre. Während im Sample eine deutlich größere Gruppe als die der zufriedenen Ruheständler eine vergleichbar positive Ruhestandsorientierung aufweist, zeichnen sich diese dadurch aus, dass es ihnen gelingt, ihr Nacherwerbsleben tatsächlich entsprechend ihrer Orientierung - und gleichsam am Leitbild „aktiven Alters“ vorbei - zu führen, was wesentlich zu der bekundeten Zufriedenheit beiträgt. Der zufriedene Ruheständler ist männlich, Akademiker mit einem überdurchschnittlichen Haushaltseinkommen und selbst gewählt aus qualifizierter Beschäftigung in den vorzeitigen Ruhestand gegangen. Die Kritiker einer produktivistischen Aktivierung des Alters sind unter den zufriedenen Ruheständlern besonders häufig vertreten.

(2) Der geschäftige Ruheständler hat hingegen ein überaus negatives Ruhestandsverständnis, mit dem eine positive Orientierung auf Facetten des Unruhestands korrespondiert. Die Ansprüche an die Sinnhaftigkeit von Alters(alltags)aktivitäten sind jedoch eher niedrig, was darin seinen Ausdruck findet, dass häufig nicht auf konkrete Tätigkeiten, sondern auf den Tatbestand des Beschäftigtseins an sich verwiesen wird: Der geschäftige Ruheständler hat „immer genug zu tun“ und ist ,jeden Tag vollauf beschäftigt“. Nichtsdestotrotz bleibt er ein Ruheständler, da der positive Orientierungsrahmen des Unruhestands nicht gelebt wird. Der geschäftige Ruheständler erweist sich mithin als Busy Talker, der sich trotz niedrigen Aktivitätsgrades und -radius als „nicht so richtig rentnertypisch“ wahrnimmt - obwohl sein Alltag ruheständlerischer kaum sein könnte. Er ist eine männliche Sozialfigur, verfügt eher über eine nicht-akademische Ausbildung sowie über ein leicht unterdurchschnittliches Haushaltseinkommen.

(3) Die verhinderte Ruheständlerin erweist sich in diesem Lichte besehen als das genaue Gegenmodell: Sie hat dem zufriedenen Ruheständler vergleichbar eine positive Ruhestandsorientierung, ohne diese jedoch praktisch umzusetzen. Stattdessen lebt sie ein hochgradig heteroproduktives, auf (mehr oder weniger signifikant) andere gerichtetes Leben als „Super-Helferin“, die hohe Belastungen (v. a. im Bereich der Betreuung und Pflege) nicht durch Einschränkungen in anderen Aktivitätsbereichen kompensiert - und durchaus damit hadert, sich nicht „abgrenzen“ zu können. Bemerkenswert ist, dass sie aus ihrem hohen Aktivitätsgrad weder eine Distinktion gegenüber weniger Aktiven ableitet noch Ansprüche an ein vergleichbares Engagement anderer formuliert. Die große Bedeutung, die sie sozialen Ehrenämtern beimisst, steht ihrer grundsätzlichen Ruhestandsorientierung insofern nicht entgegen, als damit nicht das klassische Ruhestandsmodell infrage gestellt, sondern vielmehr ein geschlechtsspezifisches, weitgehend altersunabhängiges weibliches Lebensmodell praktiziert wird. Die verhinderte Ruheständlerin ist weiblich, westdeutsch und dem nicht-akademischen Milieu zugehörig.

(4) Die Unruheständlerin trägt ihren Namen, weil ihre Vorstellung vom Nacherwerbsleben mit zentralen Elementen des Unruhestandsdispositivs übereinstimmt und sie diese Orientierung in der Alltagspraxis auch weitgehend realisiert. Sie ist durchaus familienorientiert, legt aber Wert auch auf außerfamiliäre Kontakte, auf Austausch mit anderen, Autonomie in der Partnerschaft und Unabhängigkeit von den (Enkel-)Kindern. Im Zentrum ihres Handelns steht autoproduktives, selbstbezogenes Engagement: sportliche und kulturelle Aktivitäten, Fremdsprachenerwerb und Bildungsreisen, künstlerische Hobbies und die Beschäftigung mit spirituellen Fragen sind typische Aktivitäten der Unruheständlerin, für die es - als Bildungsbürgerin - wichtig ist, nach einem Leben voller Familien- und Erwerbspflichten endlich Zeit für sich zu haben.

(5) Der/die Produktive grenzt sich in deutlicher Weise nicht nur vom Ruhestandsmodell, sondern auch vom Unruhstand ab: Wo die Unruheständlerin große Freude an autoproduktiven Aktivitäten hat, kritisiert er bzw. sie einen freizeitorientierten, allein am eigenen Wohlbefinden ausgerichteten (Un-)Ruhestand und hebt die Bedeutung und Notwendigkeit gesellschaftlichen Engagements hervor. Die Entpflichtung von der Erwerbsarbeit verpflichte zum bürgerschaftlichen Engagement, eine entsprechende gesellschaftliche Ansprache von Menschen im Nacherwerbsleben wird explizit begrüßt. Der/die Produktive stimmt nicht nur mit wesentlichen Wissenselementen des Produktivitätsdispositivs überein, das propagierte heteroproduktive Leben wird auch tatsächlich selbst geführt. Bemerkenswert ist allerdings, dass die Produktiven nicht zu den sehr Aktiven in unserem Sample zäh-

8 Stellt man beispielsweise den Ost-West-Vergleich ins Zentrum der Betrachtung, treten andere Unterschiede und Gemeinsamkeiten in den Vordergrund. 
len. ${ }^{9}$ Anders als die anderen von uns identifizierten Sozialfiguren des Nacherwerbslebens weist der/die Produktive keine augenfälligen sozialstrukturellen Merkmale auf.

(6) Die Gebremste schließlich erweist sich als Gegenpart zur verhinderten Ruheständlerin, fallen bei ihr doch gleichfalls Orientierungsrahmen und Praxis in extremer Weiser auseinander, wenn auch mit umgekehrten Vorzeichen: Wo jene durch ein hohes Maß heteroproduktiver Aktivität an einem positiv besetzten ruheständlerischen Leben gehindert wird, zeichnet sich diese durch eine ausgeprägte Unruhestandsund/oder Produktivitätsorientierung aus und entwirft von sich selbst das Bild einer „Frau der Tat". Sie hat eine abwechslungsreiche Nacherwerbsphase mit erfüllender Aktivität erwartet, die sich aus unterschiedlichen Gründen aber nicht realisieren lässt, wobei insbesondere finanzielle Prekarität, Ausgrenzungserfahrungen im ehrenamtlichen Kontext und Einschränkungen durch den Ehemann eine maßgebliche Rolle spielen. Weder Unruhestand noch Produktives Alter werden infolgedessen in gewünschter Weise gelebt, was zu großer Unzufriedenheit führt. Die Sozialfigur der Gebremsten ist weiblich und verfügt über ein deutlich unterdurchschnittliches Haushaltseinkommen. ${ }^{10}$

\section{Fazit}

Vor dem Hintergrund unserer Befunde lässt sich zunächst festhalten, dass die Perspektiven älterer Menschen auf das Nacherwerbsleben, ihre im Interview berichteten Alltagspraktiken sowie ihre Haltung zur Frage einer nachberuflichen Aktivitätsverantwortung deutlich komplexer sind, als das Dispositiv des „Produktiven Alters“ dies im Duktus der win-win-Logik nahelegt: Während eine kleine Gruppe unserer Befragten - die Produktiven - ihr Alter als ein solch harmonisches Zusammenspiel individueller und gesellschaftlicher Interessen erleben, fallen für alle anderen Älteren gesellschaftliche Produktivitätserwartungen einerseits, persönlich gewünschter bzw. realisierter Ruhestand andererseits auf die eine oder andere Weise auseinander. Von besonderer Brisanz ist diesbezüglich die - im Sinne eines durch fortgesetzte Erwerbstätigkeit gebremsten bürgerschaftlichen Engagements - de-aktivierende Wirkung finanzieller Prekarität, von der vor allem ältere Frauen betroffen sind (und wohl auch zukünftig sein werden). Die Figur des männlichen, zufriedenen Ruheständlers hingegen zeigt deutlich, dass eine nicht-aktivische (und bisweilen auch offen anti-produktivistische) Gestaltung des Nacherwerbslebens subjektiv nach wie vor gewünscht und durchaus möglich ist - wobei man sich eine entsprechende Haltung und Praxis freilich ökonomisch leisten können muss.

Auch allfällige Überlagerungen der skizzierten Altersdispositive - Ruhestand, Unruhestand, Produktives Alter - in den Orientierungen einzelner Befragter offenbaren ein eindeutig heterogeneres Positionsfeld, als wir es in der öffentlichen Neuverhandlung des Alters vorfinden. So finden sich im Sample durchaus Personen mit einer ausgesprochenen Ruhestandsorientierung, die ihre verdiente Entpflichtung und Zeitsouveränität im Alter stark machen, aus dieser dann aber die Selbstverpflichtung ableiten, ein begrenztes Quantum dieser neuen Freiheit aktiv in den Dienst der Allgemeinheit zu stellen - frei nach dem Motto: 90 \% verdienter Ruhestand, $10 \%$ gesellschaftliche Verantwortung. Durchgängig erleben wir zudem - selbst bei den Produktiven - die andauernde Prägekraft von Vorstellungen einer altersangemessenen Ruhe und des Anspruchs auf Selbstbestimmtheit in der Nacherwerbsphase. ${ }^{11}$

Schließlich offenbaren unsere Interviews eine auffällige Leerstelle und zugleich systematische Differenz gegenüber der politischen Aktivierungsprogrammatik: Das im Kontext des Produktivitätsdispositivs so zentrale Aufwertungsversprechen an die adressierten Alten - die öffentlich in Aussicht gestellte Überwindung negativer gesellschaftlicher Altersbilder und -zuschreibungen vermittels einer aktiven Demonstration von individueller Leistungsfähigkeit im Alter (vgl. van Dyk et al. 2010) - spielt, übrigens anders als die Konfrontation mit unterschiedlichsten Formen der Altersdiskriminierung, im Erfahrungshaushalt älterer Menschen keine Rolle. Die offiziell propagierte „Schönheit“ der Aktivierungsstrategie im Sinne ihres allumfassenden sozialen Nutzens ${ }^{12}$ erschließt und offenbart sich den Älteren selbst mehrheitlich - zumindest einstweilen - nicht. Der Vorstellung des produktiven Alterns als Bedingung gesellschaftlicher Anerkennung im Alter begegnen die ,jungen Alten“ je individuell mit praktischen Lebensphilosophien, die - so oder so - auf „ein Stück ,eigenes Leben“ (Beck-Gernsheim 1983) nach der Erwerbsarbeit zielen. Zumindest dem Grunde nach, und soweit die materiellen wie psycho-physischen Gegebenheiten dies im Einzelfall zulassen, stellt die „späte Freiheit" des Nacherwerbslebens insofern tatsächlich auch eine Chance zur Emanzipation dar - womöglich allerdings eine Chance auf Widerruf.

9 Umgekehrt sind viele der in großem Umfang aktiven Befragten nicht als Produktive einzustufen, da der Orientierungsrahmen des Produktiven Alters von ihnen nicht geteilt oder sogar explizit kritisiert wird.

10 Der im Vergleich mit anderen Interviewten mittlere Aktivitätsgrad der Gebremsten geht daher auch nicht vorrangig auf selbst gewähltes Engagement, sondern auf die finanziell notwendige Aufnahme bzw. Beibehaltung geringfügiger Beschäftigung zurück, sodass hier in gewissem Sinne eine „Zwangsaktivierung" vorliegt.

11 Insofern decken sich unsere Befunde mit denen einer anderen Jenaer Arbeitsgruppe zu den biografischen "Lebenskraftkalkülen“ qualifizierter Erwerbstätiger (vgl. Behr/Hänel 2013)

12 "All generations stand to gain something important from policy changes which enable and motivate older persons to become and remain more active." (European Commission 1999, S. 22) 


\section{LITERATUR}

Beck-Gernsheim, E. (1983): Vom „Dasein für andere“ zum Anspruch auf ein Stück „eigenes Leben“: Individualisierungsprozesse im weiblichen Lebenszusammenhang, in: Soziale Welt 34 (3), S. 307-340

Behr, M./Hänel, A. (2013): Höher qualifizierte Angestellte als Lebenskraftkalkulierer - Eine Herausforderung für die betriebliche Alterspolitik, in: WSI-Mitteilungen 66 (2), S. 98-106, http://www.boeckler.de/wsimit_2013_02_behr.pdf Boudiny, K. (2012): "Active ageing": from empty rhetoric to effective policy tool, in: Ageing and Society, DOI: http://dx.doi.org/10.1017/ S0144686X1200030X

Bröckling, U./Krasmann, S. (2010): Ni méthode, ni approche. Zur Forschungsperspektive der Gouvernementalitätsstudien - mit einem Seitenblick auf Konvergenzen und Divergenzen zur Diskursforschung, in: Angermüller, J./van Dyk S. (Hrsg.): Diskursanalyse meets Gouvernementalitätsforschung, Frankfurt/ New York, S. 23-42

Bröckling, U./Krasmann, S./Lemke, T. (Hrsg.) (2000): Gouvernementalität der Gegenwart, Studien zur Ökonomisierung des Sozialen, Frankfurt a. M. Denninger, T./van Dyk, S./Lessenich, S./Richter, A. (2010): Die Regierung des Alter(n)s. Analysen im Spannungsfeld von Diskurs, Dispositiv und Disposition in: Angermüller, J./van Dyk, S. (Hrsg.): Diskursanalyse meets Gouvernementalitätsforschung, Frankfurt/New York, S. 207-235

Denninger, T./van Dyk, S./Lessenich, S./Richter, A. (2012): Vom "verdienten Ru hestand" zum „Alterskraftunternehmer"? Bilder des Alter(n)s im gesellschaftlichen Wandel nach dem Systemumbruch, in: Best, H./Holtmann, E. (Hrsg.): Aufbruch der entsicherten Gesellschaft, Frankfurt/New York, S. 369-387

Denninger, T./van Dyk, S./Lessenich, S./Richter, A. (2013): Leben im Ruhestand. Zur Neuverhandlung des Alters in der Aktivgesellschaft, Bielefeld (im Erscheinen)

Deutscher Bundestag (2006): Fünfter Bericht zur Lage der älteren Generation in der Bundesrepublik Deutschland - Potenziale des Alters in Wirtschaft und Gesellschaft - Der Beitrag älterer Menschen zum Zusammenhalt der Generationen, Drucksache 16/2190, Berlin

Deutscher Bundestag (2010): Sechster Bericht zur Lage der älteren Generation in der Bundesrepublik Deutschland - Altersbilder in der Gesellschaft, Drucksache $17 / 3815$, Berlin

Dyk, S. van /Lessenich, S. (Hrsg.) (2009): Die jungen Alten. Analysen einer neuen Sozialfigur, Frankfurt/New York

Dyk, S. van /Lessenich, S./Denninger, T./Richter, A. (2010): Die "Aufwertung" des Alters. Eine gesellschaftliche Farce, in: Mittelweg 3619 (5), S. 15-33

Ekerdt, D. J. (1986): The Busy Ethic: Moral Continuity Between Work and Retirement, in: The Gerontologist 26 (3), S. 239-244

European Commission (1999): Towards a Europe for all Ages. Promoting Prosperity and Intergenerational Solidarity, COM(1999) 221 final, Brussels

Graefe, S./Lessenich, S. (2012): Rechtfertigungsordnungen des Alter(n)s, in: Soziale Welt 63 (4), S. 299-315

Hajer, M. A. (1997): Ökologische Modernisierung als Sprachspiel. Eine institutionell-konstruktivistische Perspektive zum Umweltdiskurs und zum institutionellen Wandel, in: Soziale Welt 48 (2), S. 107-132

Kohli, M. (1985): Die Institutionalisierung des Lebenslaufs. Historische Befunde und theoretische Argumente, in: Kölner Zeitschrift für Soziologie und Sozialpsychologie 37 (1), S. 1-29

Krasmann, S./Volkmer, M. (Hrsg.) (2007): Michel Foucaults „Geschichte der Gouvernementalität" in den Sozialwissenschaften, Internationale Beiträge, Bielefeld

Lessenich, S. (2008): Die Neuerfindung des Sozialen. Der Sozialstaat im flexiblen Kapitalismus, Bielefeld
Lessenich, S. (2013): Alles muss raus: Die politische Logik des Potenzials, in: WSI-Mitteilungen 66 (2), S. 76, http://www.boeckler.de/wsimit_2013_02_kommentar.pdf

Rosenmayr, L. (1983): Die späte Freiheit. Das Alter, ein Stück bewußt gelebten Lebens, Berlin

Schimany, P. (2003): Die Alterung der Gesellschaft. Ursachen und Folgen des demographischen Umbruchs, Frankfurt/New York.

Tews, H. P. (1990): Neue und alte Aspekte des Strukturwandels des Alters, in: WSI-Mitteilungen 43 (8), S. 478-491

Walker, A. (2002): A strategy for active ageing, in: International Social Security Review 55 (1), S. $121-139$

World Bank (1994): Averting the old age crisis: politics to protect the old and promote growth, Washington D.C.

\section{AUTOREN}

SILKE VAN DYK, Dr., Akademische Rätin am Institut für Soziologie der Friedrich-Schiller-Universität Jena. Arbeitsschwerpunkte: Soziologie des Alter(n)s und der Demografie, Wohlfahrtsstaatsforschung, Gesellschaftstheorie, Dis kurstheorie und empirische Diskursforschung.

silke.vandyk@uni-jena.de

STEPHAN LESSENICH, Prof. Dr., Leiter des Arbeitsbereichs Gesellschaftsvergleich und Sozialer Wandel am Institut für Soziologie der Friedrich-SchillerUniversität Jena. Arbeitsschwerpunkte: Wohlfahrtsstaatsforschung, Soziologie des Alter(n)s, Soziologie des sozialen Wandels.

stephan.lessenich@uni-jena.de

TINA DENNINGER, Dipl.-Soz., Akademische Rätin am Institut für Soziologie der Ludwig-Maximilians-Universität München. Promotion zum Thema "Schönheit im Alter". Arbeitsschwerpunkte: Körpersoziologie, Soziologie des Alter(n)s, Diskursforschung.

tina.denninger@soziologie.uni-muenchen.de

ANNA RICHTER, Dipl.-Pol., Wissenschaftliche Mitarbeiterin am Institut für Sozialwesen der Universität Kassel, Fachgebiet Lebenslagen und Altern. Promotion zum Thema "Altern und Geschlecht in biografischen Erzählungen ostdeutscher Frauen“. Arbeitsschwerpunkte: Intersektionalität und Diversity, Soziologie des Alter(n)s, Ostdeutschlandforschung, Diskurs- und Biografieforschung.

@anna.richter@uni-kassel.de 